\title{
Tests of pulse shape discrimination with EJ299-33 plastic scintillator for use in portable spectroscopy
}

\section{Angus Comrie* and Andy Buffler}

Department of Physics, University of Cape Town, Rondebosch, 7700, South Africa

E-mail: angus.comrieduct.ac.za, andy.bufflereuct.ac.za

\section{Ricky Smit}

iThemba LABS, Somerset West, 7129, South Africa

E-mail: smitetlabs.ac.za

\section{Heinrich Wörtche}

INCAS ${ }^{3}$, Dr. Nassaulaan 9. 9400 AT Assen, The Netherlands

E-mail: HeinrichWoertchedincas3.eu

\begin{abstract}
The pulse shape discrimination capbability of the newly available EJ299-33 plastic scintillator is compared with that of the widely-used EJ301 liquid scintillator, using a digital implementation of the charge comparison algorithm. Typical pulse shapes for neutrons and gamma-rays produced by an Am-Be radioisotropic source are fitted to an analytical model, in order to determine the time constants and relative weightings of the fast, medium and slow decay components of scintillation. These pulse shapes are compared to those generated by a Monte-Carlo simulation, utilising the Geant4 toolkit.
\end{abstract}

Technology and Instrumentation in Particle Physics 2014,

2-6 June, 2014

Amsterdam, the Netherlands

${ }^{*}$ Speaker. 


\section{Introduction}

The need for a compact neutron detector has grown in recent years, mainly for deployment in aircraft and spacecraft, for minerals exploration, personal and workplace dosimetry and in security, particularly the detection of special nuclear materials. Since neutrons are seldom present without gamma-rays, any useful detector is required to be able to discriminate between the two radiation types. Furthermore, for dosimetry in the upper atmosphere and in space, neutrons in the energy range 1-100 MeV need to be measured.

The light output of certain organic scintillators display both fast and slow decay components, related to the specific energy loss of the recoiling charged particle by the Birks relation [1]. Pulse shape discrimination (PSD), established in the 1950s [2] and developed through the 1960s [3], allows the identification of different types of charged particles in these scintillator detectors by means of the characteristics of the scintillation decay. Liquid organic scintillators such as the nearlyequivalent NE213, BC501A and EJ301 persist as popular detector-types for this application. Two recent technological developments are assisting the employment of hydrocarbon-based detectors together with PSD in non-laboratory environments. Firstly, the development of solid ("plastic") polyvinyltoluene-based detectors [4, 5] which exhibit PSD capability, such as the EJ299-33 scintillator characterized in this work, removes the toxic and fire hazards associated with most liquid scintillators. Secondly, the emergence of digital data acquisition and processing systems allows pulse shape discrimination to be implemented and dynamically optimised in software form across a wide energy range [6-8], or implemented on specialised hardware, such as field programmable gate arrays (FPGAs) or digital signal processors (DSPs). In this work, we present a characterization of the newly developed EJ299-33 scintillator, which is currently being used in the construction of a compact scintillation detector, consisting of a plastic scintillator, coupled to silicon photomultipliers (SiPMs) and read out by an FPGA-based acquisition system.

\section{Experimental setup}

The detector used for the characterization of the EJ299-33 plastic consisted of cylindrical scintillator (51 mm diameter x $51 \mathrm{~mm}$ ) optically coupled to an ETL 9214 12-stage photomultiplier tube and base, supplied by Scionix, and operated at a negative bias of $950 \mathrm{~V}$. For comparative measurements, an encapsulated EJ301 liquid scintillator of the same dimensions was used. Signals from the PMT anode were digitised by a CAEN Vx1761 10 bit digitizer, operating at 4 GS/s with a peak-to-peak range of 1 volt. Custom-built software was used to record data for offline analyses. Neutrons and gamma-rays were provided via a $2 \mathrm{GBq}{ }^{241} \mathrm{Am}-{ }^{9} \mathrm{Be}(\mathrm{Am}-\mathrm{Be})$ radioisotopic source, placed $20 \mathrm{~cm}$ away from the detector. Light output spectra were calibrated using gamma-rays from ${ }^{137} \mathrm{Cs},{ }^{22} \mathrm{Na}$ and Am-Be radioisotopic sources.

\section{Pulse shape discrimination}

\subsection{Timing}

In order to perform effective PSD, a consistent signal start time $t_{0}$ must be defined in a way which minimises effects due to variations in pulse noise, baseline, amplitude and shape. A digital 
constant fraction discriminator (dCFD) filter was applied to each signal. The filtered signal $v$ takes the form of

$$
v_{i}=\sum_{j=1}^{N} f V_{i-j}-V_{i-j-D}
$$

where $V$ is the unfiltered signal, $N$ is the filter length, $D$ is the filter offset and $f$ is the filter fraction [9]. The filtered signal is the difference between two moving average filters, with the first filter scaled by $f$, and the second filter offset by $D$ samples. This produces a bipolar pulse, and $t_{0}$ was taken as the time at which the filtered pulse switches polarity, minus a constant offset of $25 \mathrm{~ns}$, in order to include the entire pulse in further calculations.

\subsection{Charge comparison}

For each recorded event, a parameter $Q_{L}$ was calculated by integrating the digitised signal over a $250 \mathrm{~ns}$ time interval $\left(t_{L}\right)$, starting from $t_{0}$. The baseline, calculated as an average of 300 samples preceding the leading edge of each pulse, was subtracted to avoid effects of voltage drift. The gamma-rays from ${ }^{22} \mathrm{Na},{ }^{137} \mathrm{C}$ and $\mathrm{Am}-\mathrm{Be}$ sources were used for scaling $Q_{L}$ to a light output parameter $L$ in units of $\mathrm{MeV}$ electron equivalent $\left(\mathrm{MeV}_{\mathrm{ee}}\right)$. The relationship between $Q_{L}$ and $L$ was found to be linear over the energy range of the calibration sources for both EJ299-33 and EJ301 scintillators, and was assumed to be linear over the energy range of the measurements. A second parameter $Q_{S}$ was calculated by integrating the pulse over a shorter time interval $\left(t_{S}\right)$. A pulse shape parameter $S$ was then defined as

$$
S=k \frac{Q_{S}}{Q_{L}}+C,
$$

which is the basis of a charge comparison method of PSD. Constants $k$ and $C$ were chosen in order to appropriately scale and offset $S$. Signals arising from gamma-ray interactions with the detector have a reduced slow decay component in comparison to those arising from neutron interactions. Therefore, a larger proportion of light output occurs within the shorter time interval in gammaray events, leading to higher values of $S$ when compared to neutron events of a similar light output. Statistical fluctuations in pulse shape lead to Gaussian distributions of $S$ for gamma-ray and neutron events with equal values of $Q_{L}$.

\section{Characterization of EJ299-33 plastic scintillator}

\subsection{Light output and pulse shape discrimination}

Fig. 1 shows distributions of events as a function of parameters $L$ and $S$ for events in (a) EJ301 and (b) EJ299-33, when exposed to neutrons and gamma-rays from the Am-Be source. The loci associated with recoiling electrons from Compton scattering events and protons recoiling from n-p elastic scattering are well separated over the full range of $L$ for EJ301, while the loci for EJ299-33 overlap significantly at lower $L$. The gamma-ray events result mainly from the detection of (a) 4.43 $\mathrm{MeV}$ gamma rays from the de-excitation of ${ }^{12} \mathrm{C}$ in the $\mathrm{Am}-\mathrm{Be}$ source and (b) $4.43 \mathrm{MeV}$ and 2.23 $\mathrm{MeV}$ gamma-rays produced by ${ }^{12} \mathrm{C}\left(\mathrm{n}, \mathrm{n}\right.$ ') and ${ }^{1} \mathrm{H}(\mathrm{n}, \gamma) \mathrm{d}$ interactions with polyethylene surrounding the detector. The dotted lines show cuts to separate events associated with neutrons and gammarays. The projections of these selections onto the $L$-axis are shown in Fig. 2. The dashed lines in 

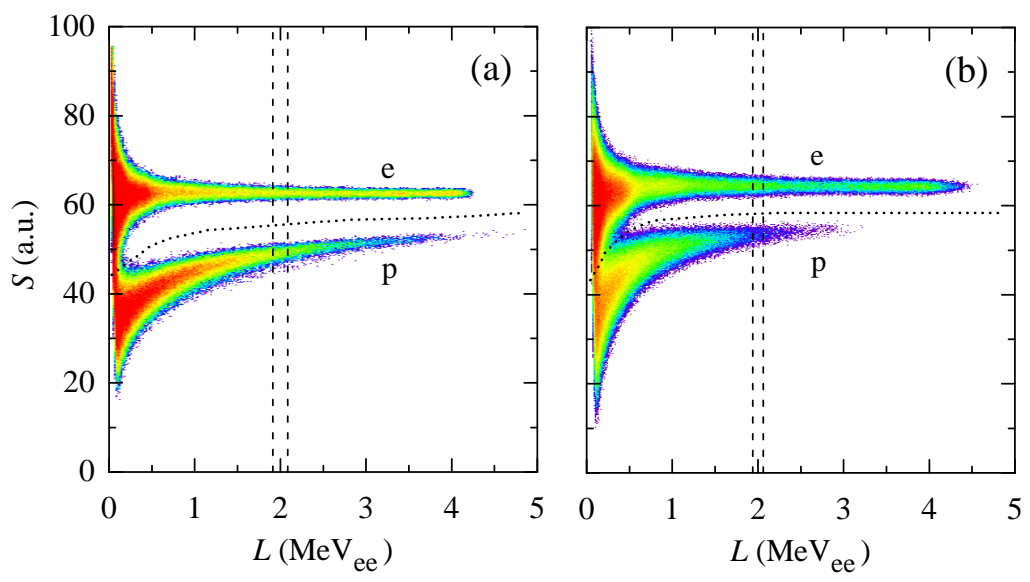

Figure 1: Counts as a function of light output parameter $L$ and pulse shape parameter $S$ for events in (a) EJ301 and (b) EJ299-33, when exposed to neutrons and gamma-rays from the Am-Be source. Loci associated with recoiling electrons (e) and protons (p) are indicated. The dotted lines indicate the cuts used to separate neutron and gamma-ray events and the dashed lines the cut used to select events in the range $L=$ 1.9 to $2.1 \mathrm{MeV}_{\mathrm{ee}}$.
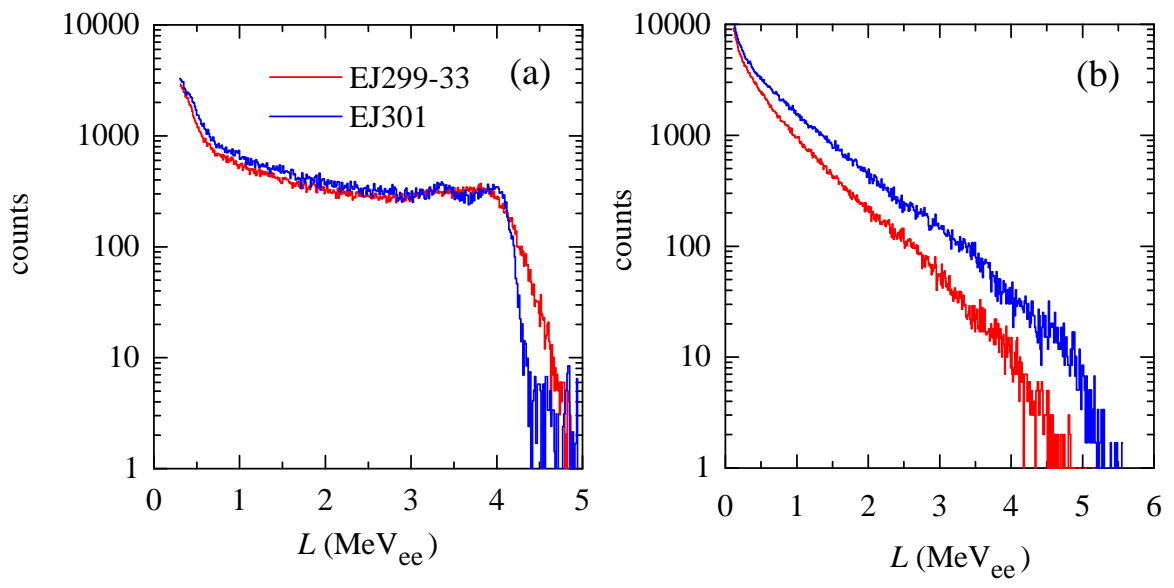

Figure 2: Light output $L$ spectra from EJ299-33 and EJ301, for (a) gamma-rays and (b) neutrons from AmBe, as selected by the cuts shown in Fig. 1.

Fig. 1 indicate a cut to select events in the range $L=1.9$ to $2.1 \mathrm{MeV}_{\mathrm{ee}}$, which are projected onto the $S$-axis in Fig. 3(a). The light output spectra in Fig. 2(a) show that EJ299-33 has a noticeably reduced energy resolution when compared to EJ301. Fig. 2(b) shows that the relative amount of light produced by protons in EJ299-33 is smaller to that of protons in EJ301, and the efficiency for neutron detection is also slightly reduced.

A figure of merit (FoM) is a useful means to provide a quantitative measurement of the separation between the distributions of $S$ for two pulse classes [10]. If these distributions are Gaussian in form, it is reasonable to define a FoM in terms of the mean $\mu$ and full width at half maximum 

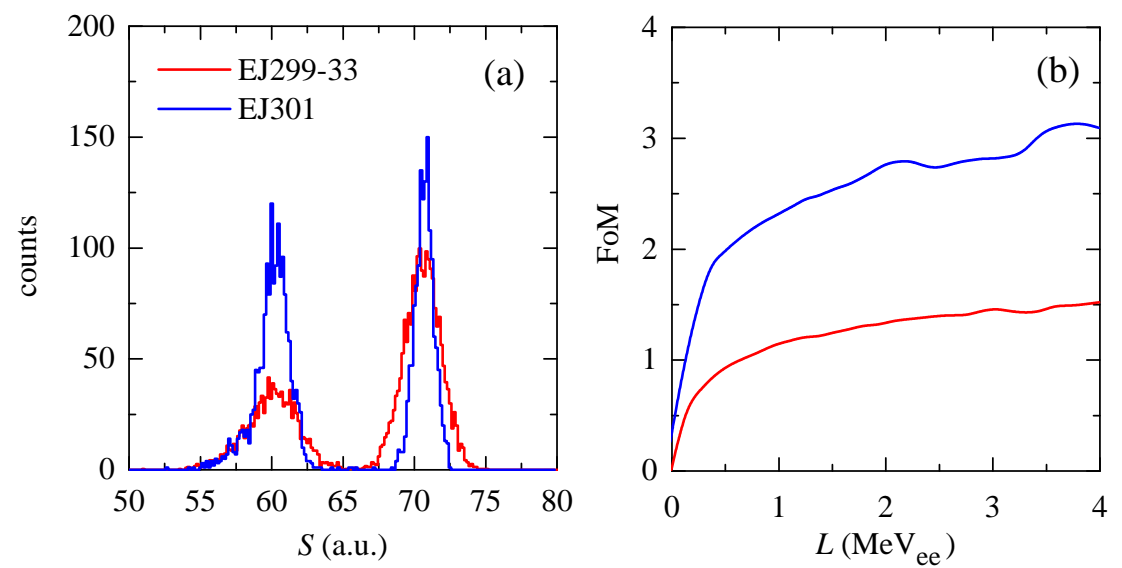

Figure 3: (a) Counts versus shape parameter $S$ for events in the range of $L=1.9$ to $2.1 \mathrm{MeV}_{\text {ee }}$, as selected by the cuts shown in Fig. 1. The scale and offset of the EJ299-33 histogram have been adjusted in order to align the distributions; (b) Figure of merit versus light output parameter $L$ for events in EJ301 and EJ299-33.
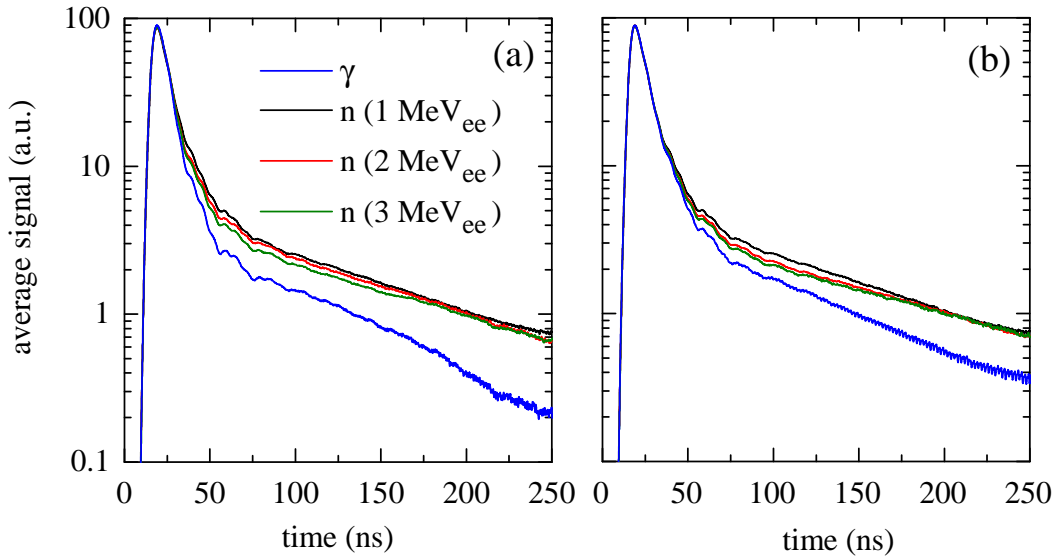

Figure 4: Average signals produced by by $L=1 \mathrm{MeV}_{\mathrm{ee}}$ gamma-rays and $L=1,2$ and $3 \mathrm{MeV}_{\text {ee }}$ neutrons in (a) EJ301 and (b) EJ299-33, normalised to the same total integral.

(FWHM) of the two distributions:

$$
\mathrm{FoM}=\frac{\left|\mu_{e}-\mu_{p}\right|}{F W H M_{e}+F W H M_{p}} .
$$

The short integration time $t_{S}$ was chosen in order optimise the FoM, with typical values of $30 \mathrm{~ns}$ and $50 \mathrm{~ns}$ for EJ301 and EJ299-33 respectively. Figure 3(b) shows FoM as a function of $L$ for the two scintillators. The FoM for EJ301 remains above 1.0 for energies above $100 \mathrm{keV}$. The plastic scintillator displays significantly inferior FoMs across the entire light output range of the experiment, with FoM dropping below 1.0 for energies below $600 \mathrm{keV}$.

\subsection{Pulse shapes}

Fig. 4 shows the average signals produced by $L=1 \mathrm{MeV}_{\text {ee }}$ gamma-ray events and $L=1,2$ and $3 \mathrm{MeV}_{\text {ee }}$ neutron events in (a) EJ301 and (b) EJ299-33, calculated by summing over a number 


\begin{tabular}{|c|c|c|c|c|}
\hline (a) EJ301 & \multicolumn{4}{|c|}{$\tau_{1}=4.32 \pm 0.10 \mathrm{~ns} ; \tau_{2}=11.17 \pm 0.58 \mathrm{~ns} ; \tau_{3}=106.5 \pm 2.5 \mathrm{~ns}$} \\
\hline Variable & gamma-rays & $\mathrm{n}\left(L=1 \mathrm{MeV}_{\mathrm{ee}}\right)$ & $\mathrm{n}\left(L=2 \mathrm{MeV}_{\mathrm{ee}}\right)$ & $\mathrm{n}\left(L=3 \mathrm{MeV}_{\mathrm{ee}}\right)$ \\
\hline$A$ & 0.674 & 0.428 & 0.556 & 0.581 \\
\hline$B$ & 0.261 & 0.380 & 0.337 & 0.323 \\
\hline$C$ & 0.053 & 0.111 & 0.095 & 0.086 \\
\hline$R^{2}$ & 0.999 & 0.997 & 0.999 & 0.998 \\
\hline (b) EJ299-33 & \multicolumn{4}{|c|}{ 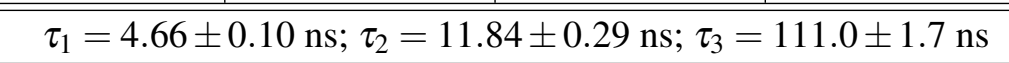 } \\
\hline Variable & gamma-rays & $\mathrm{n}\left(L=1 \mathrm{MeV}_{\mathrm{ee}}\right)$ & $\mathrm{n}\left(L=2 \mathrm{MeV}_{\mathrm{ee}}\right)$ & $\mathrm{n}\left(L=3 \mathrm{MeV}_{\mathrm{ee}}\right)$ \\
\hline$A$ & 0.507 & 0.470 & 0.493 & 0.497 \\
\hline$B$ & 0.421 & 0.415 & 0.405 & 0.408 \\
\hline$C$ & 0.061 & 0.100 & 0.089 & 0.084 \\
\hline$R^{2}$ & 0.999 & 0.999 & 0.998 & 0.998 \\
\hline
\end{tabular}

Table 1: Fitted parameters of pulse shapes for (a) EJ301 and (b) EJ299-33, for $L=1 \mathrm{MeV}_{\text {ee }}$ gamma-rays, and $L=1,2$ and $3 \mathrm{MeV}_{\text {ee }}$ neutrons, as well as the coefficient of determination $R^{2}$ for each fit.

of events within narrow regions of $L$ and normalised by their total integrals. There is noticeable variation in the pulse shape as a function of $L$ for neutron pulses, which results in the bending of the proton loci (see Fig. 1). The tails of the signals in Fig. 4 follow an exponential decay function of the form

$$
f(t)=A \exp \left(-\frac{t}{\tau_{1}}\right)+B \exp \left(-\frac{t}{\tau_{2}}\right)+C \exp \left(-\frac{t}{\tau_{3}}\right),
$$

where $\tau_{1}, \tau_{2}$ and $\tau_{3}$ are the time constants of fast, medium and slow decay components respectively, and $A, B$ and $C$ are their relative weightings. Equation 4.2 was used to fit the average gamma-ray signal, in order to determine the time constants for EJ301 and EJ299-33. These time constants were then used to fit the average signals from $L=1,2$ and $3 \mathrm{MeV}_{\text {ee }}$ neutron events, in order to determine the relative weightings. The results are given in Table 1 . The coefficient of determination $R^{2}$ is close to unity for each fit, indicating excellent agreement with the data.

The two scintillators have similar time constants, but the relative weightings differ. In particular, fit parameter $B$ is much larger in EJ299-33. The difference between the values of fit parameter $A$ for neutron and gamma-ray events in EJ299-33 is far smaller than the difference in EJ301, which results in the inferior PSD performance described in Section 4.1.

\subsection{Comparison with Geant4 simulation}

A new feature added to version 10.0 of the Geant 4 Monte-Carlo simulation toolkit [11] is the ability to simulate the time-dependent light output of scintillators. This feature has been previously validated for the simulation of a standard liquid scintillator [12]. In this work, the experimental measurements described in Sections 4.1 and 4.2 are compared to the output of a Geant 4 simulation of an EJ299-33 scintillator, coated with a reflective paint and coupled to an ideal PMT. The parameters in Table 1 were used as input to the simulation. Parameters related to total light output response of the scintillator to protons were taken from Lawrence et. al. [13]. Fig. 5(a) compares the simulated and measured average signals produced by gamma-rays and neutrons from ${ }^{22} \mathrm{Na}$ and 

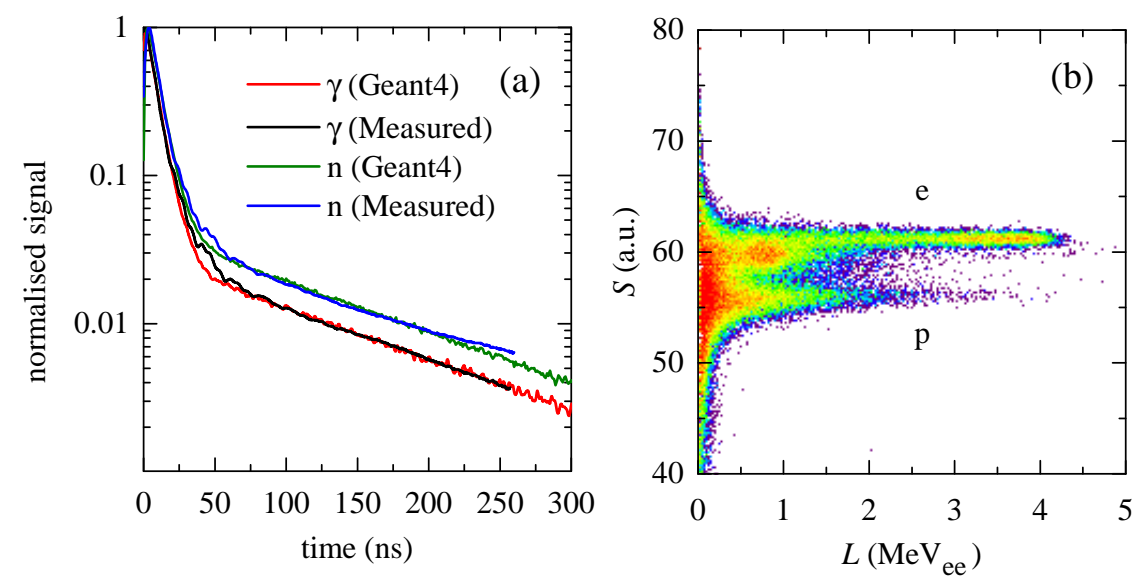

Figure 5: (a) Simulated and measured average signals produced by gamma-rays from ${ }^{22} \mathrm{Na}$ and neutrons from AmBe in EJ299-33. The signals have been normalised by their pulse height. (b) Counts as a function of light output parameter $L$ and pulse shape parameter $S$ for simulated events in EJ299-33.

AmBe sources respectively. There is good agreement between measured and simulated signals for both gamma-ray and neutron signals. Currently, only two decay components can be simulated by the Geant 4 toolkit. This limitation is most likely responsible for the small discrepancy between simulated and measured signals in Fig. 5(a) around $t=50 \mathrm{~ns}$, along with the presence of reflections in the PMT.

The use of a simulation capable of reproducing pulse shapes allows the investigation of the effect of changes to detector geometry, wrapping or light collection mechanisms, all of which influence pulse shape discrimination capability. Fig. 5(b) shows the distribution of simulated events as a function of the light output parameter $L$, and the pulse shape parameter $S$, showing a clear separation between neutron and gamma-ray events at higher energies, and a similar behaviour to the measured distribution shown in Fig. 1(b). The absence of bending of the proton locus in Fig. 5 (b) is due to a limitation of the Geant4 toolkit, which currently does not allow for an energydependent weighting of the fast and slow scintillation decay components. Future adaptations of the simulation will address the limitations in the Geant 4 toolkit mentioned above and will include the effect of photomultiplier response, particularly for use with silicon photomultipliers, which convolute the scintillator response with an additional slow $(\tau \sim 100 \mathrm{~ns})$ decay.

\section{Conclusion}

A comparison of detectors based on the EJ299-33 plastic scintillator and EJ301 liquid scintillator shows that, while the plastic scintillator displays PSD capability, FoM values are roughly half of those for the liquid scintillator. This inhibits efficient separation of neutron and gamma-ray events at low energies. An investigation into the pulse shapes of the two scintillators shows that the weighting of the fast decay component of scintillation does not differ between proton and electron interactions as significantly in EJ299-33 when compared to EJ301, leading to a lower FoM.

The simulation of pulse shapes using the Geant 4 toolkit provides a quantitative method of predicting PSD capability, and is capable of reproducing the pulse shapes and PSD capability of 
the EJ299-33 scintillator. Future measurements of response of the plastic scintillator to neutrons with energies of up to $100 \mathrm{MeV}$, produced by a cyclotron facility, will characterise the behaviour of EJ299-33 over a wider energy range, and determine its feasability for use in high-altitude dosimetry.

\section{References}

[1] J. Birks, Scintillations from organic crystals: specific fluorescence and relative response to different radiations, Proceedings of the Physical Society. Section A 64 (1951) 874.

[2] G. Wright, Scintillation decay times of organic crystals, Proceedings of the Physical Society. Section B 69 (1956) 358.

[3] F. Brooks, Development of organic scintillators, Nucl. Instr. and Meth. 162 (1979) 477.

[4] N. Zaitseva, B. L. Rupert, I. Pawełczak, A. Glenn, H. P. Martinez, L. Carman, M. Faust, N. Cherepy, and S. Payne, Plastic scintillators with efficient neutron/gamma pulse shape discrimination, Nucl. Instr. and Meth. A 668 (2012) 88.

[5] D. Cester, G. Nebbia, L. Stevanato, F. Pino, and G. Viesti, Experimental tests of the new plastic scintillator with pulse shape discrimination capabilities EJ-299-33, Nucl. Instr. and Meth. A 735 (2014) 202.

[6] B. Esposito, Y. Kaschuck, A. Rizzo, L. Bertalot, and A. Pensa, Digital pulse shape discrimination in organic scintillators for fusion applications, Nucl. Instr. and Meth. A 518 (2004) 626.

[7] Y. Kaschuck and B. Esposito, Neutron/ $\gamma$-ray digital pulse shape discrimination with organic scintillators, Nucl. Instr. and Meth. A 551 (2005) 420.

[8] A. C. Comrie, A. Buffler, F. D. Smit, and H. J. Wörtche, Digital neutron/gamma discrimination with an organic scintillator at energies between $1 \mathrm{MeV}$ and $100 \mathrm{MeV}, \mathrm{Nucl}$. Instr. Meth. A 772 (2015) 43.

[9] H. Peng, P. Olcott, A. Foudray, and C. Levin, Evaluation of free-running ADCs for high resolution pet data acquisition, in Nuclear Science Symposium Conference Record, 2007. NSS'07. IEEE, vol. 5, p. 3328, IEEE, 2007.

[10] C. Brient, C. Nelson, and R. Young, Pulse shape analyzer for fast neutron-gamma ray discrimination, Nucl. Instr. and Meth. 98 (1972) 329.

[11] S. Agostinelli, J. Allison, K. a. Amako, J. Apostolakis, H. Araujo, P. Arce, M. Asai, D. Axen, S. Banerjee, G. Barrand, et al., Geant4 - a simulation toolkit, Nucl. Instr. and Meth. A 506 (2003) 250.

[12] Z. S. Hartwig and P. Gumplinger, Simulating response functions and pulse shape discrimination for organic scintillation detectors with Geant4, Nucl. Instr. and Meth. A 737 (2014) 155.

[13] C. C. Lawrence, M. Febbraro, T. N. Massey, M. Flaska, F. Becchetti, and S. A. Pozzi, Neutron response characterization for an EJ299-33 plastic scintillation detector, Nucl. Instr. and Meth. A 759 (2014) 16. 